\title{
Effect of Acarbose on Long-Term Prognosis in Acute Coronary Syndromes Patients with Newly Diagnosed Impaired Glucose Tolerance
}

\author{
Peng Yun, ${ }^{1}$ Ai-ming Du, ${ }^{2}$ Xue-jun Chen, ${ }^{2}$ Jing-cheng Liu, ${ }^{3}$ and Hu Xiao ${ }^{3}$ \\ ${ }^{1}$ Department of Internal Medicine, School of Clinical Medicine, Yangtze University, Jingzhou, Hubei 434000, China \\ ${ }^{2}$ Department of Cardiology, Central Hospital of Jingzhou City, Hubei 434001, China \\ ${ }^{3}$ Department of Endocrinology, The First Affiliated Hospital of Yangtze University, Jingzhou, Hubei 434000, China \\ Correspondence should be addressed to Hu Xiao; 449838290@qq.com
}

Received 23 April 2015; Accepted 30 June 2015

Academic Editor: Yunzhou Dong

Copyright (C) 2016 Peng Yun et al. This is an open access article distributed under the Creative Commons Attribution License, which permits unrestricted use, distribution, and reproduction in any medium, provided the original work is properly cited.

Objective. To investigate the effect of acarbose therapy on the long-term prognosis of patients with acute coronary syndromes (ACS) complicating newly diagnosed impaired glucose tolerance (IGT). Methodology. 135 patients hospitalized for ACS who had been newly diagnosed with IGT were randomly assigned to acarbose group ( $150 \mathrm{mg} /$ day, $n=67$ ) or control group (no acarbose, $n=68$ ). All cases in each group were given the same elementary treatment. Mean follow-up was 2.3 years. The incidence of major adverse cardiovascular event (MACE) and carotid intima-middle thickness (CIMT) were statistically analyzed. Results. During the mean follow-up of 2.3 years, the risk of recurrent MACE in acarbose group was decreased significantly compared with that in control group $(26.67 \%$ versus $46.88 \%, P<0.05)$; at the same time, thickening of the CIMT was significantly slower than the control group $((1.28 \pm 0.42) \mathrm{mm}$ versus $(1.51 \pm 0.64) \mathrm{mm}, P<0.05)$. Conclusions. Acarbose can effectively reduce the risk of MACE in ACS patients with newly diagnosed IGT, simultaneously retarding the progression of carotid intima-media thickness.

\section{Introduction}

As gradual steps into aging society and changes of lifestyle, the prevalence of impaired glycometabolism and coronary artery disease (CAD) increases rapidly in China. Impaired glucose tolerance (IGT) has been regarded as a prediabetic state in which postprandial blood glucose is between normal glucose tolerance and overt diabetes mellitus (DM). It is well known that IGT is an independent risk factor of cardiovascular events (CV) and cardiovascular-associated mortality [1]. Acarbose can effectively reduce postprandial blood glucose and the progression from IGT to Type 2 DM (T2DM). STOPNIDDM study had proved that acarbose therapy reduced the risk of any CV by $49 \%$, of an acute myocardial infarction (AMI) by $91 \%$, and of developing hypertension by $34 \%$ in IGT patients [2]. Emerging evidence suggested a high prevalence of unrecognized IGT and/or DM in patients admitted to hospital with acute coronary syndrome (ACS) [3]. Furthermore, such dysglycaemia has been shown to be associated with an increase in cardiovascular mortality. Therefore, we have reason to postulate that acarbose treatment may reduce major adverse cardiovascular event (MACE) in patients with ACS complicating impaired glycometabolism. No related research has been reported so far. Thus, the goal of present study is to determine whether acarbose can reduce the risk of recurrent MACE in ACS patients with newly diagnosed IGT.

\section{Research Design and Methods}

2.1. Study Patients. From March 2010 to August 2013, we randomly selected 426 cases of patients who were hospitalized in our two hospitals due to ACS. ACS was diagnosed by the presence of acute ischemic symptoms lasting $\geqslant 20$ min within $48 \mathrm{~h}$ before admission to hospital and electrocardiographic changes consistent with ACS [4]. Acute myocardial infarction (AMI) was diagnosed when creatine kinase-MB levels increased to at least twice the upper limit of normal or when troponin T levels were $>0.1 \mathrm{ng} / \mathrm{mL}$. Patients without AMI 
TABLE 1: Baseline characteristics of study patients and frequencies of medication usage before admission.

\begin{tabular}{|c|c|c|c|}
\hline & $\begin{array}{l}\text { Control group } \\
\quad(n=68)\end{array}$ & $\begin{array}{l}\text { Acarbose group } \\
(n=67)\end{array}$ & $P$ value \\
\hline \multicolumn{4}{|l|}{ Basic characteristic } \\
\hline Age, year & $61.62 \pm 4.58$ & $62.24 \pm 5.16$ & 0.461 \\
\hline Male, $n(\%)$ & $42(61.76)$ & $39(58.21)$ & 0.673 \\
\hline Smoking, $n(\%)$ & $31(45.59)$ & $28(41.79)$ & 0.657 \\
\hline AMI, $n(\%)$ & $43(63.24)$ & $46(68.66)$ & 0.506 \\
\hline Hypertension, $n(\%)$ & $46(67.65)$ & $45(67.16)$ & 0.637 \\
\hline LVEF, \% & $52.13 \pm 4.81$ & $51.74 \pm 5.25$ & 0.653 \\
\hline Revascularization (PCI/CABG), $n(\%)$ & $38(55.88)$ & $41(61.19)$ & 0.531 \\
\hline $\mathrm{BMI}, \mathrm{kg} / \mathrm{m}^{2}$ & $25.82 \pm 2.45$ & $26.05 \pm 3.24$ & 0.427 \\
\hline \multicolumn{4}{|l|}{ Medications } \\
\hline$\beta$-blocker, $n(\%)$ & $16(23.53)$ & $13(19.40)$ & 0.559 \\
\hline ACE-I/ARB, $n(\%)$ & $47(69.12)$ & $45(67.16)$ & 0.808 \\
\hline $\mathrm{CCB}, n(\%)$ & $18(26.47)$ & $20(29.85)$ & 0.662 \\
\hline Statin, $n(\%)$ & $63(92.65)$ & $61(91.04)$ & 0.734 \\
\hline Aspirin, $n(\%)$ & $64(94.12)$ & $62(92.54)$ & 0.713 \\
\hline
\end{tabular}

AMI: acute myocardial infarction; PCI: percutaneous coronary intervention; CABG: coronary artery bypass grafting; BMI: body mass index; ACE-I: angiotensin-converting enzyme inhibitor; ARB: angiotensin II receptor blocker; CCB: calcium channel blocker.

were considered to have unstable angina pectoris (all cases confirmed by percutaneous or computed tomography coronary angiography). Exclusion criteria were as follows: (1) cardiogenic shock or pulmonary edema (Killip classification $\geqslant$ II) at admission, (2) history of diabetes, (3) history of hepatic diseases or/and renal dysfunction (serum creatinine level $>2 \mathrm{mg} / \mathrm{dL}$ ), (4) severe gastrointestinal disease or malignant tumors, and (5) female patients given sex hormone replacement therapy. All cases underwent the standard $75 \mathrm{~g}$ oral glucose tolerance test (OGTT) two weeks after admission, and we used the WHO criteria to classify the OGTT results. IGT was defined as having a fasting plasma glucose (FPG) $<6.1 \mathrm{mmol} / \mathrm{L}$ and a postprandial glucose level at $120 \mathrm{~min}$ after the glucose load $(2 \mathrm{hPG}) \geqslant 7.8 \mathrm{mmol} / \mathrm{L}$ but $<11.1 \mathrm{mmol} / \mathrm{L} .135$ cases were newly diagnosed with IGT. The present study followed Helsinki principle which was reviewed and approved by the Ethics Committee; all patients and their families provided informed consent.

2.2. Methods. 135 IGT patients were randomly allocated, using random numbers generated by a computer, into the following two groups: the control group (68 cases) and acarbose group (67 cases). Each group was given standard medical therapy of CAD (including nitrate medications, ACE-I/ARB, $\beta$-blockers, statins, and antiplatelet drugs). Acarbose group was given additional acarbose (Bayer Pharmaceutical Co., Germany, three times/day, $25 \mathrm{mg} /$ time start, and gradually increasing the amount to $50 \mathrm{mg} /$ time in 2 weeks) on the base of fundamental treatment. Carotid intima-media thickness (CIMT) was measured using Siemens SEQUOIA512 ultrasonography, taking the point under $1 \mathrm{~cm}$ of carotid sinus as detection point and accounting the average of the left and right CIMT as the results. The carotid IMT was measured at baseline, and follow-up of all subjects, $\triangle$ CIMT, indicated the changes in CIMT. All patients were guided to take diet and exercise therapy, and having outpatient clinic or telephone follow-up for 1.0-4.5 years, the mean follow-up was 2.3 years. Incidence of MACE (including fatal cardiovascular events, nonfatal reinfarction, new-onset angina, cerebral stroke, and severe heart failure) was recorded.

2.3. Statistical Analysis. Statistical analysis was performed using SPSS 13.0. Continuous variables were expressed as mean and standard deviation, and categorical variables were expressed as using numbers and percentages. Comparisons among the two groups were performed using Student's $t$ test and paired $t$-test for continuous variables and $\chi^{2}$ test for categorical variables. Values for $P$ less than 0.05 were accepted as statistically significant.

\section{Results}

3.1. Clinical Background. The clinical profile of the subjects was shown in Table 1. There was no significant difference in age, gender, profiles for traditional risk factors, and past medication history between the two groups $(P>0.05)$. Of the total of 135 cases in the average follow-up of 2.3 years, 11 patients dropped out during the study which was $8.15 \%$ of all subjects ( 7 patients in the acarbose group: 5 male and 2 female; 4 patients in the control group: 2 male and 2 female). The cause was severe abdominal distension and diarrhea for 6 cases in the acarbose group, or lost for 1 case in acarbose group and 4 cases in control group, respectively; the incidence of serious gastrointestinal adverse reactions between the two groups was statistically significant $\left(\chi^{2}=6.373, P=0.012\right)$.

3.2. The Risk of Recurrent MACE. Recurrent MACE was observed in the 124 cases that completed the study. Table 2 
TABLE 2: MACE among the study patients in the mean 2.3-year follow-up $(n, \%)$.

\begin{tabular}{lcc}
\hline & $\begin{array}{c}\text { Control } \\
\text { group } \\
(n=64)\end{array}$ & $\begin{array}{c}\text { Acarbose } \\
\text { group } \\
(n=60)\end{array}$ \\
\hline Cardiovascular death, $n(\%)$ & $5(7.81)$ & $3(5.00)$ \\
Nonfatal reinfarction, $n(\%)$ & $7(10.94)$ & $2(3.33)$ \\
New-onset angina, $n(\%)$ & $9(14.06)$ & $5(8.33)$ \\
Cerebral stroke, $n(\%)$ & $4(6.25)$ & $2(3.33)$ \\
Severe heart failure, $n(\%)$ & $5(7.81)$ & $4(6.67)$ \\
Total MACE, $n(\%)$ & $30(46.88)$ & $16(26.67)$ \\
\hline
\end{tabular}

MACE: major adverse cardiovascular events.

demonstrated the numbers of MACE among subjects in the mean 2.3-year follow-up. The incidence of total MACE in the acarbose group and control group was $26.67 \%$ and $46.88 \%$, respectively; there was significant difference between the two groups $\left(\chi^{2}=5.420, P=0.020\right)$. In total MACE, the death of 3 patients in acarbose group and 5 patients in control group was due to cardiovascular events, and the cardiovascular caused mortality between the two groups was of no significant difference $\left(\chi^{2}=0.406, P=0.524\right)$. However, the incidence of the secondary end-point events (nonfatal reinfarction, newonset angina, cerebral stroke, and severe heart failure) of the acarbose group was prominently lower than that of control group (21.67\% versus $39.06 \%, \chi^{2}=4.410, P=0.036$ ).

\subsection{Laboratory and Ultrasonography Examination. Table 3} shows the level of biochemical indicators, CIMT, blood pressure, and BMI of the two groups before and after treatment. All the indexes were not significantly different between the two groups before treatment $(P>0.05)$. After treatment, $2 \mathrm{hPG}, \mathrm{HbAlc}$, CIMT, and $\triangle \mathrm{CIMT}$ of the acarbose group were significantly lower than control group $(t$ value was 8.731, $6.198,2.440$, and 18.622 , resp., $P<0.05$ or 0.01 ), while FPG, TC, TG, LDL-C, systolic pressure, diastolic pressure, and BMI had no statistical significance between the two groups after treatment ( $t$ value was $1.528,0.242,1.102,0.214,1.201,0.883$, and 1.866, resp., $P>0.05)$.

\section{Discussion}

Postprandial hyperglycemia is a hallmark characteristic in individuals with IGT and early T2DM and has been established as a key pathophysiological component of the mechanism underlying the development of diabetic complications [5]. Fluctuations in glucose levels following a meal are strongly associated with micro- and macrovascular complications not only in patients with T2DM, but also in individuals with IGT [6]. The risk for developing CAD or other MACE was increased almost threefold in subjects with IGT compared to people with normal glucose tolerance [7]. Kataoka et al. [8] found that multibranch coronary artery lesion which was common in T2DM patients had already emerged in the IGT stage. Recent study indicated that IGT was an independent risk factor for AMI and simultaneously increased the risk of recurrent CV after AMI [9]. Furthermore, Kitada et al. [10] showed that postprandial blood glucose above $8.9 \mathrm{mmol} / \mathrm{L}$ would increase the risk of recurrent MACE nearly one-fold in patients with AMI. There is a high prevalence of unrecognized dysglycaemia in patients with ACS; the present study found that $31.69 \%$ of the patients with ACS were newly diagnosed IGT; it was similar to previous researches $[3,11]$. Thus, early intervention to IGT in ACS patients with the aim of reducing recurrent MACE should be anticipated.

IGT is a prediabetic state; several management strategies have been proposed for this early stage of dysglycaemia, including lifestyle modification and pharmacotherapies (e.g., acarbose, metformin, and thiazolidinediones) [12-14]. Although lifestyle modification is a vital part of dysglycaemia management, it is often insufficient to maintain long-term glycaemic control. Given that acarbose has a relatively modest efficacy of blood glucose lowering and can be administered to patients with heart failure and mild to moderate renal insufficiency, acarbose is safer than other drugs mentioned above for glucose management in ACS patients, because management of glucose levels within a given range and with minimal risk of hypoglycemia is recommended for the treatment of hyperglycemia in patients with ACS [15]. In the present study, $2 \mathrm{hPG}$ and HbAlc of the acarbose group significantly reduced compared with those of control group $(P<0.01)$; this result was not unexpected. The starting dose of acarbose was semiquantity and gradually increased to normal dose ( $50 \mathrm{mg} /$ time, three times/day), so only 6 patients $(8.96 \%)$ withdrew from the trial due to severe gastrointestinal side effects. The other patients were well tolerated and with no hypoglycemia.

Previous studies $[2,16]$ had suggested that acarbose was useful in reducing the risk of MACE in patients with IGT; its cardiovascular protective effect might be due to the reduction of postprandial hyperglycemia and glucose variability, increased insulin sensitivity, induction of moderate weight loss, restoration of endothelial function, and so forth [17]. In the above mechanisms, the improvement of vascular endothelial function is reasonably important. Endothelial dysfunction plays an important role in the development of atherosclerosis and predicts $\mathrm{CV}$ outcomes independent of conventional CV risk factors [18]. Although the mechanism by which postprandial hyperglycemia induces endothelial dysfunction is not fully understood, oxidative stressmediated disruptions in nitric oxide homeostasis are implicated as key role [19]. Endothelium-derived nitric oxide (NO) is one of the most potent known endogenous vasodilators and it plays an important role in the control of coronary blood flow by regulating vascular tone. Kato et al. [20] found acarbose improved postprandial endothelial function by improvement of postprandial hyperglycemia in patients with newly diagnosed T2DM. This notion had been recently reinforced by the finding that 24 weeks of acarbose monotherapy in newly diagnosed patients with T2DM was associated with increased levels of both fasting and postprandial glucagonlike peptide 1 (GLP-1), NO levels, and nitric oxide synthase (NOS) activity [21]. Thus, acarbose seemed to favorably affect endothelial function in the coronary arteries and contributed 
TABLE 3: Biochemical indicator level, CIMT, BMI, and blood pressure of two groups between pre- and posttreatment (mean \pm SD).

\begin{tabular}{|c|c|c|c|c|}
\hline & \multicolumn{2}{|c|}{ Control group } & \multicolumn{2}{|c|}{ Acarbose group } \\
\hline & $\begin{array}{c}\text { Pretreatment } \\
\quad n=68\end{array}$ & $\begin{array}{c}\text { Posttreatment } \\
n=64\end{array}$ & $\begin{array}{c}\text { Pretreatment } \\
\quad n=67\end{array}$ & $\begin{array}{c}\text { Posttreatment } \\
n=60\end{array}$ \\
\hline $\mathrm{FPG}, \mathrm{mmol} / \mathrm{L}$ & $5.84 \pm 0.33$ & $5.95 \pm 0.54$ & $5.92 \pm 0.42$ & $5.78 \pm 0.69$ \\
\hline $2 \mathrm{hPG}, \mathrm{mmol} / \mathrm{L}$ & $8.76 \pm 0.49$ & $9.46 \pm 1.22$ & $8.98 \pm 0.54$ & $7.64 \pm 1.08^{\#, \mathbf{\Lambda}}$ \\
\hline HbAlc, \% & $6.28 \pm 0.23$ & $6.36 \pm 0.51$ & $6.30 \pm 0.28$ & $5.92 \pm 0.24^{\#, \mathbf{\Lambda}}$ \\
\hline Systolic, mmHg & $148.54 \pm 8.63$ & $140.27 \pm 6.25^{*}$ & $150.16 \pm 10.38$ & $138.82 \pm 7.14^{\boldsymbol{\Lambda}}$ \\
\hline Diastolic, $\mathrm{mmHg}$ & $93.26 \pm 5.34$ & $88.51 \pm 4.62^{*}$ & $94.13 \pm 7.18$ & $87.73 \pm 5.18^{\wedge}$ \\
\hline $\mathrm{TC}, \mathrm{mmol} / \mathrm{L}$ & $6.24 \pm 1.03$ & $5.06 \pm 0.96^{*}$ & $6.38 \pm 1.35$ & $5.03 \pm 0.87^{\mathbf{\Lambda}}$ \\
\hline $\mathrm{TG}, \mathrm{mmol} / \mathrm{L}$ & $2.36 \pm 0.58$ & $1.74 \pm 0.52^{*}$ & $2.45 \pm 0.62$ & $1.65 \pm 0.38^{\mathbf{\Lambda}}$ \\
\hline LDL-C, mmol/L & $3.07 \pm 0.66$ & $2.56 \pm 0.52^{*}$ & $3.18 \pm 0.75$ & $2.58 \pm 0.58^{\triangle}$ \\
\hline BMI, kg/m ${ }^{2}$ & $25.86 \pm 2.45$ & $25.64 \pm 2.75$ & $26.02 \pm 3.47$ & $24.65 \pm 3.13^{\wedge}$ \\
\hline CIMT, mm & $1.23 \pm 0.46$ & $1.49 \pm 0.54^{*}$ & $1.24 \pm 0.52$ & $1.28 \pm 0.41^{\#}$ \\
\hline$\Delta \mathrm{CIMT}, \mathrm{mm}$ & \multicolumn{2}{|c|}{$0.22 \pm 0.07$} & \multicolumn{2}{|c|}{$0.05 \pm 0.02^{\#}$} \\
\hline
\end{tabular}

${ }^{*} P<0.05$, versus the control group before treatment; ${ }^{\#} P<0.05$, versus the control group after treatment; ${ }^{\mathbf{\Delta}} P<0.05$, versus the acarbose group before treatment.

to an improved long-term prognosis. The present study showed that acarbose could effectively reduce total risk of MACE in ACS patients with newly diagnosed IGT $(P<0.05)$. This cardiovascular benefit mostly owed to the reduction of secondary endpoint events $(P<0.05)$, while the decline of cardiovascular disease mortality was not statistically significant $(P>0.05)$, which might be related to not long enough follow-up time and not enough number of patients enrolled.

The absolute value and change of CIMT are both indirect indicators of coronary atherosclerosis and independent predictors of long-term CV [22, 23]. Previous study had confirmed that acarbose could slow the progression of CIMT in patients with IGT or T2DM [24,25], and Koyasu et al. [26] found that acarbose also could retard CIMT thickness and plaque formation in CAD patients with newly diagnosed IGT. The present study showed that acarbose slowed the progression of CIMT in ACS patients with IGT, which also indirectly suggested that acarbose delayed the development of coronary atherosclerosis in patients with IGT. In the present study, BMI in acarbose group had a distinctly downtrend compared with the control group $(P=0.064)$; it suggested that moderate loss of weight may be one of the possible reasons for the cardiovascular benefit.

In summary, the present study indicates that acarbose can effectively and safely retard the CIMT thickness and reduce the risk of recurrent MACE in ACS patients with newly diagnosed IGT. Therefore, acarbose can improve the prognosis of these patients.

\section{Conflict of Interests}

The authors declared that they have no conflict of interests in this work.

\section{Authors' Contribution}

Peng Yun and Ai-ming Du contributed equally to this work.

\section{References}

[1] U. Zeymer, "Cardiovascular benefits of acarbose in impaired glucose tolerance and type 2 diabetes," International Journal of Cardiology, vol. 107, no. 1, pp. 11-20, 2006.

[2] J.-L. Chiasson, R. G. Josse, R. Gomis, M. Hanefeld, A. Karasik, and M. Laakso, "Acarbose treatment and the risk of cardiovascular disease and hypertension inpatients with impaired glucose tolerance: the STOP-NIDDM trial," Journal of the American Medical Association, vol. 290, no. 4, pp. 486-494, 2003.

[3] A. A. Tahrani, J. Geen, F. W. F. Hanna et al., "Predicting dysglycaemia in patients under investigation for acute coronary syndrome," QJM, vol. 104, no. 3, pp. 231-236, 2011.

[4] R. S. Wright, J. L. Anderson, C. D. Adams et al., "2011 ACCF/ AHA focused update of the guidelines for the management of patients with unstable angina/non-ST-elevation myocardial infarction (Updating the 2007 Guideline): a report of the American College of cardiology foundation/American heart association task force on practice guidelines," Circulation, vol. 123, no. 18, pp. 2022-2060, 2011.

[5] E. Standl and O. Schnell, "Alpha-glucosidase inhibitors 2012cardiovascular considerations and trial evaluation," Diabetes and Vascular Disease Research, vol. 9, no. 3, pp. 163-169, 2012.

[6] R. J. Heine, B. Balkau, A. Ceriello, S. Del Prato, E. S. Horton, and M.-R. Taskinen, "What does postprandial hyperglycaemia mean?” Diabetic Medicine, vol. 21, no. 3, pp. 208-213, 2004.

[7] E. Standl, O. Schnell, and A. Ceriello, "Postprandial hyperglycemia and glycemic variability. Should we care?" Diabetes Care, vol. 34, no. 2, pp. S120-S127, 2011.

[8] Y. Kataoka, S. Yasuda, I. Morii, Y. Otsuka, A. Kawamura, and S. Miyazaki, "Quantitative coronary angiographic studies of patients with angina pectoris and impaired glucose tolerance," Diabetes Care, vol. 28, no. 9, pp. 2217-2222, 2005.

[9] K. Tamita, M. Katayama, T. Takagi et al., "Newly diagnosed glucose intolerance and prognosis after acute myocardial infarction: comparison of post-challenge versus fasting glucose concentrations," Heart, vol. 98, no. 11, pp. 848-854, 2012.

[10] S. Kitada, Y. Otsuka, N. Kokubu et al., "Post-load hyperglycemia as an important predictor of long-term adverse cardiac events 
after acute myocardial infarction: a scientific study," Cardiovascular Diabetology, vol. 9, no. 1, article 75, 2010.

[11] C. Hage, P. Lundman, L. Rydén, and L. Mellbin, "Fasting glucose, $\mathrm{HbAlc}$, or oral glucose tolerance testing for the detection of glucose abnormalities in patients with acute coronary syndromes," European Journal of Preventive Cardiology, vol. 20, no. 4, pp. 549-554, 2013.

[12] M. Hanefeld, A. Karasik, C. Koehler, T. Westermeier, and J.-L. Chiasson, "Metabolic syndrome and its single traits as risk factors for diabetes in people with impaired glucose tolerance: the STOP-NIDDM trial," Diabetes and Vascular Disease Research, vol. 6, no. 1, pp. 32-37, 2009.

[13] Diabetes Prevention Program Research Group, "10-Year followup of diabetes incidence and weight loss in the Diabetes Prevention Program outcomes study,' The Lancet, vol. 374, no. 9702, pp. 1677-1686, 2009.

[14] R. A. DeFronzo, M. A. Banerji, G. A. Bray et al., "Actos now for the prevention of diabetes (ACT NOW) study," BMC Endocrine Disorders, vol. 9, article 17, 2009.

[15] M. Kosiborod and P. Deedwania, "An overview of glycemic control in the coronary care unit with recommendations for clinical management," Journal of Diabetes Science and Technology, vol. 3, no. 6, pp. 1342-1351, 2009.

[16] M. Hanefeld, F. Schaper, and C. Koehler, "Effect of acarbose on vascular disease in patients with abnormal glucose tolerance," Cardiovascular Drugs and Therapy, vol. 22, no. 3, pp. 225-231, 2008.

[17] E. Standl, M. J. Theodorakis, M. Erbach, O. Schnell, and J. Tuomilehto, "On the potential of acarbose to reduce cardiovascular disease," Cardiovascular Diabetology, vol. 13, article 81, 2014.

[18] M. Veerasamy, A. Bagnall, D. Neely, J. Allen, H. Sinclair, and V. Kunadian, "Endothelial dysfunction and coronary artery disease: a state of the art review," Cardiology in Review, vol. 23, no. 3, pp. 119-129, 2015.

[19] E. Mah and R. S. Bruno, "Postprandial hyperglycemia on vascular endothelial function: mechanisms and consequences," Nutrition Research, vol. 32, no. 10, pp. 727-740, 2012.

[20] T. Kato, T. Inoue, and K. Node, "Postprandial endothelial dysfunction in subjects with new-onset type 2 diabetes: an acarbose and nateglinide comparative study," Cardiovascular Diabetology, vol. 9, article 12, 2010.

[21] M.-Y. Zheng, J.-H. Yang, C.-Y. Shan et al., "Effects of 24week treatment with acarbose on glucagon-like peptide 1 in newly diagnosed type 2 diabetic patients: a preliminary report," Cardiovascular Diabetology, vol. 12, article 73, 2013.

[22] D. H. O’Leary, J. F. Polak, R. A. Kronmal, T. A. Manolio, G. L. Burke, and S. K. Wolfson Jr., "Carotid-artery intima and media thickness as a risk factor for myocardial infarction and stroke in older adults," The New England Journal of Medicine, vol. 340, no. 1, pp. 14-22, 1999.

[23] M. W. Lorenz, H. S. Markus, M. L. Bots, M. Rosvall, and M. Sitzer, "Prediction of clinical cardiovascular events with carotid intima-media thickness: a systematic review and metaanalysis," Circulation, vol. 115, no. 4, pp. 459-467, 2007.

[24] M. Hanefeld, J. L. Chiasson, C. Koehler, E. Henkel, F. Schaper, and T. Temelkova-Kurktschiev, "Acarbose slows progression of intima-media thickness of the carotid arteries in subjects with impaired glucose tolerance," Stroke, vol. 35, no. 5, pp. 1073-1078, 2004.

[25] T. Oyama, A. Saiki, K. Endoh et al., "Effect of acarbose, an alpha-glucosidase inhibitor, on serum lipoprotein lipase mass levels and common carotid artery intima-media thickness in type 2 diabetes mellitus treated by sulfonylurea," Journal of Atherosclerosis and Thrombosis, vol. 15, no. 3, pp. 154-159, 2008.

[26] M. Koyasu, H. Ishii, M. Watarai et al., "Impact of acarbose on carotid intima-media thickness in patients with newly diagnosed impaired glucose tolerance or mild type 2 diabetes mellitus: a one-year, prospective, randomized, open-label, parallelgroup study in Japanese adults with established coronary artery disease," Clinical Therapeutics, vol. 32, no. 9, pp. 1610-1617, 2010. 


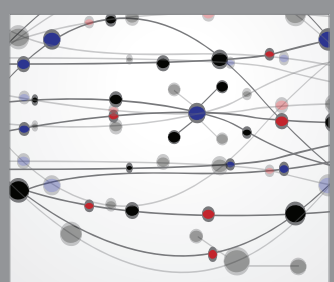

The Scientific World Journal
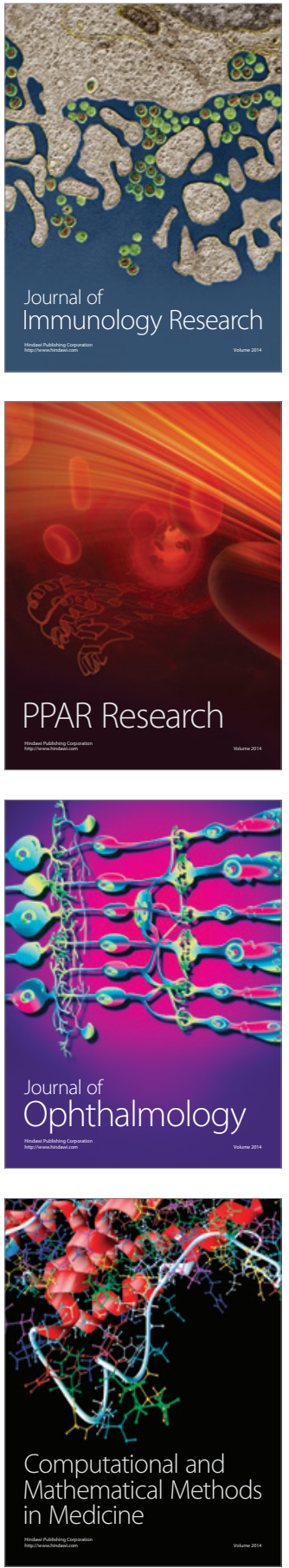

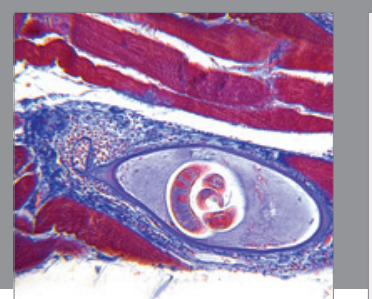

Gastroenterology Research and Practice

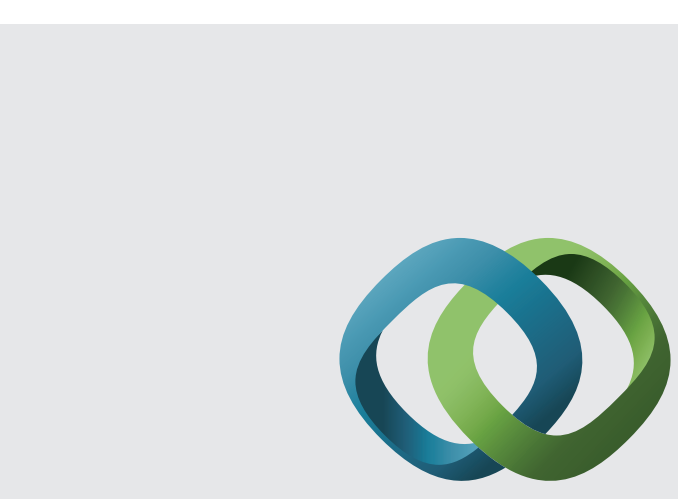

\section{Hindawi}

Submit your manuscripts at

http://www.hindawi.com
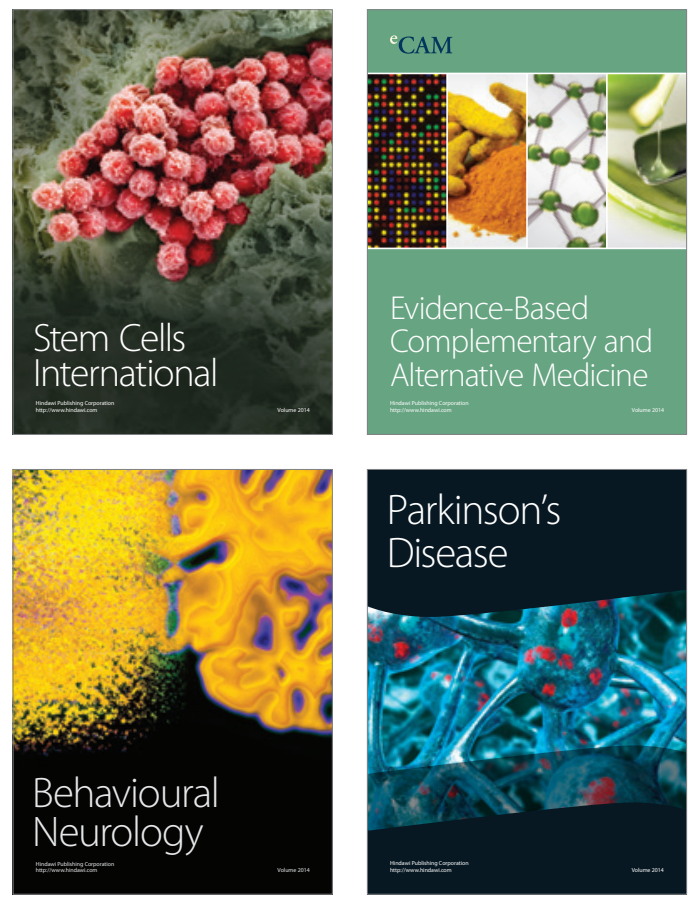
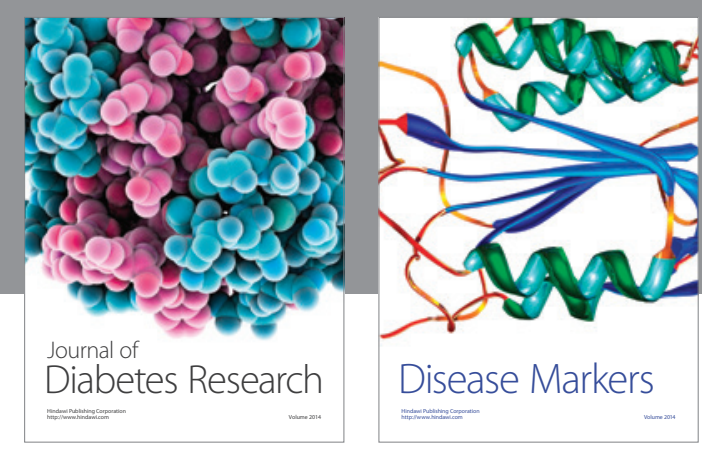

Disease Markers
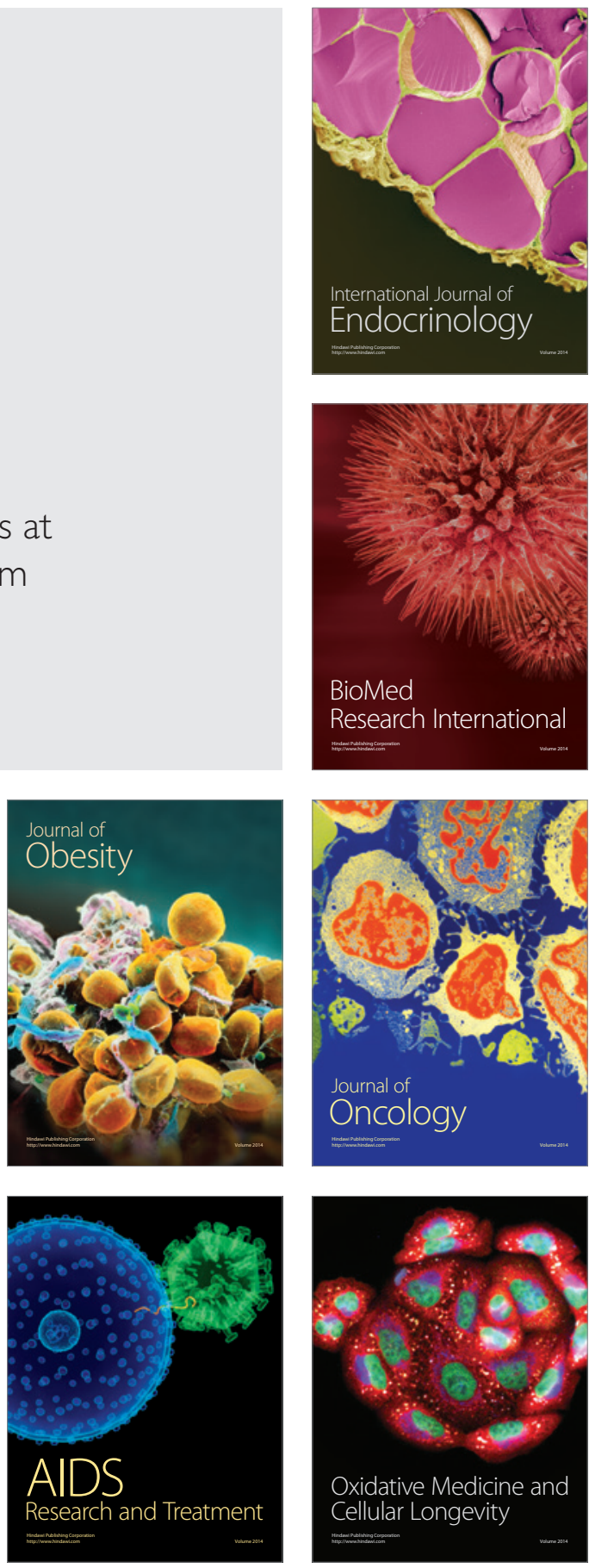